\title{
OBSERVATORIO
}

\section{PSICOLOGÍA Y SOCIOLOGÍA DE LA INFORMACIÓN: UNA NECESIDAD PRÁCTICA Y TEÓRICA}

\author{
Francisco-Javier García-Marco
}

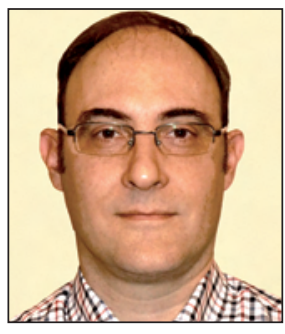

Francisco-Javier García-Marco es doctor en filosofía y letras desde 1994 y profesor titular de universidad del Área de Biblioteconomía y Documentación de la Universidad de Zaragoza desde 1996. Investiga, enseña y publica sobre las aplicaciones de las nuevas tecnologías de la información a la documentación científica, la difusión de la cultura y la gestión social, así como sobre la teoría de la documentación, la organización del conocimiento, el tratamiento y recuperación de la información, los sistemas de información histórica y la historia social de los mudéjares aragoneses. Colabora en asociaciones, eventos y publicaciones científicas nacionales e internacionales, y es director de los Encuentros Internacionales sobre Sistemas de Información y Documentación y de las revistas Scire e Ibersid.

Universidad de Zaragoza Facultad de Filosofía y Letras Pedro Cerbuna, 12. 50009 Zaragoza jgarcia@unizar.es

\section{Resumen}

Se examina el papel de la psicología y la sociología de la información en las cinco principales áreas interdisciplinares de las ciencias de la documentación: la gestión de unidades y servicios de información y documentación, la ingeniería de sistemas de información y documentación, la recuperación de la información, la estructura y dinámica de la información y la teoría de la ciencia de la información. En los últimos años, a medida que el impacto del cambio tecnológico se ha ido asumiendo y normalizando, la perspectiva psicológica y social ha ido cobrado renovada importancia en la actividad académica y profesional de la disciplina.

\section{Palabras clave}

Ciencia de la información, Ciencias de la documentación, Aspectos interdisciplinares, Psicología de la información, Sociología de la información, Ciencias cognitivas, Ciencias sociales.

\section{Title: Information psychology and sociology: a practical and theoretical need}

\begin{abstract}
The role of the psychology and sociology of information is discussed in the five main interdisciplinary areas of library and information science: information services management, information systems engineering, information retrieval, information structure and dynamics, and information science theory. In recent years, after the impact of technological change has been progressively accommodated, psychological and sociological perspectives are assuming a growing importance in our discipline.
\end{abstract}

\section{Keywords}

Library and information science, Interdisciplinary aspects, Psychology of information, Sociology of information, Social sciences, Cognitive sciences.

García-Marco, Francisco-Javier. "Psicología y sociología de la información: una necesidad práctica y teórica". El profesional de la información, 2011, enero-febrero, v. 20, n. 1, pp. 5-9.

DOI: 10.3145/epi.2011.ene.01

\section{Importancia de los aspectos psicológicos y sociales en la ciencia de la información}

La oportunidad de dedicar el tema central de El profesional de la información a la psicología y la sociología de la información no parece necesitar mucha discusión. La información -tal y como la tratan las profesiones de la información- es un fenómeno eminentemente humano, y por ello es imposible estudiar la información humana o realizar una actividad fundamentada -sea en la práctica profesional, en 
la investigación o en la reflexión teórica- en este campo sin atender a sus fundamentos psicológicos y sociales.

$\mathrm{Y}$, sin embargo, a pesar de ser un tema necesario -e incluso omnipresente- es también un tema difícil, pues se puede abordar de muchas maneras: desde la mera opinión, la ideología, la reflexión más o menos sistemática y coherente (humanidades), o por supuesto desde la búsqueda de evidencia observable y compartida que caracteriza la investigación científica (ciencias humanas y, dentro de éstas, las sociales). Además, todas estas incursiones se pueden hacer $-\mathrm{y}$ se hacen- desde múltiples perspectivas y paradigmas.

Es importante señalar antes que, en los últimos años, a medida que una gran parte de la investigación nuclear del área se deslizaba hacia las ingenierías -la recuperación de la información es cada vez más una disciplina informática en manos de grandes empresas como Google-, la perspectiva psicológica y social ha cobrado renovada importancia en la actividad académica y profesional de la disciplina. Un trabajo publicado recientemente por Cronin (2008) tuvo el acierto de etiquetar ese movimiento como The sociological turn in information science.

Gran parte de los conocimientos psicológicos y sociales que se manejan en la ciencia y las profesiones de la información son importados de las humanidades y las ciencias humanas, y luego concretados en modelos específicos que son los que inspiran la práctica y la investigación del área.

Esta realidad se puede observar en los cinco grandes grupos de temas que constituyen las ciencias de la documentación: 1) la gestión de unidades y servicios de información y documentación, 2) la ingeniería de sistemas de información y documentación, 3) la recuperación de la información -modelos matemáticos, metadatos y organización del conocimiento, como aproximaciones alternativas y muchas veces complementarias-, 4) la estructura y dinámica de la información -bibliometría y uso de la información, fundamentalmente-, y 5) la teoría de la ciencia de la información.

En todos ellos la psicología y la ciencia social ocupan un lugar importante que examinaremos someramente a continuación caso por caso.

\section{Gestión de unidades y servicios de información y documentación}

La gestión de unidades de información constituye -junto con la catalogación e indización- la parte central de la actividad profesional en el área. La información y la documentación es un campo de la actividad humana que se ha profesionalizado en nichos destacados como los archivos, la gestión de documentos, las bibliotecas, la documentación de medios, la documentación científica y otras documentaciones especializadas, el comercio del libro, la evaluación de la ciencia, y, cada vez más, en la gestión de contenidos.

En ese sentido, la psicología, la sociología y otras ciencias sociales proporcionan instrumentos para abordar dichos nichos de forma más eficaz y eficiente. Campos enteros como la gestión de los recursos humanos, el marketing, la dirección, la educación de usuarios, y otros muchos, son abordados con modelos y técnicas de las ciencias sociales, fundamentalmente de las aplicadas.

La gestión de recursos humanos, el marketing y las relaciones con los usuarios han recibido una atención reducida en la investigación aunque suelen estar presentes en los planes de estudios desde que se instauró la licenciatura en documentación y, por supuesto, en la práctica profesional. En los últimos años han proliferado los cursos de formación permanente en este campo, que había sido descuidado a favor de las técnicas de tratamiento y las tecnologías.

En un contexto en el que los metadatos cada vez más se añaden a los propios documentos en los procesos de creación y distribución tanto en archivos como en bibliotecas $-\mathrm{y}$, en general, de creciente automatización de todos los procesos técnicos-, el futuro de buena parte de la red de bibliotecas y archivos reside precisamente en su carácter de proximidad en el servicio a los usuarios. Por ello, la relación con el usuario está cobrando cada vez mayor importancia.

Esta es probablemente la razón del despegue del campo de la formación de usuarios -devenido en alfabetización informacional o alfin- y en el que sí se está produciendo no sólo práctica sino también un importante caudal de investigación, realizada por grupos importantes en España como el que lidera María Pinto-Molina desde la Universidad de Granada.

\section{La relación con el usuario está cobrando cada vez mayor importancia, razón del despegue de la alfin}

Otro campo interdisciplinar de gran importancia en el ámbito de la gestión, aunque muy discutido, es el de la gestión del conocimiento. En la definición del mismo hay una gran confusión, pues existe una tendencia a convertir el término en un sinónimo atractivo y sugerente de especialidades concretas -informática aplicada a la gestión organizacional, gestión de contenidos, gestión de competencias...-de diferentes disciplinas. Sin embargo, debe entenderse más bien, desde nuestro punto de vista, como un área transdisciplinar de integración de funciones muy importantes para la vida de las organizaciones que muchas veces discurren separadas y que forman parte del ciclo del conocimiento: creación e innovación; documentación de productos, servicios e ideas; publicación y difusión primaria; memoria corporativa y tecnologías de la información; difusión secundaria, educación y transferencia... La gestión del conocimiento solamente se puede entender abordando tres aspectos: la estructura y la dinámica organizacional en su conjunto, la inserción de los diferentes grupos en ese contexto, y la interacción de los individuos entre ellos y con el resto del sistema estudiado. Así planteado, está claro que aborda problemas de índole psicológica y social.

\section{Ingeniería de sistemas de información}

No existe una separación entre tecnología y ciencias humanas, especialmente en el campo de las tecnologías de la información y la comunicación, que han surgido de forma es- 
trechamente unidas a la ciencia cognitiva y al desarrollo de disciplinas sociales como la ciencias de la comunicación. El esfuerzo tecnológico ha permitido financiar proyectos científicos en el campo de la psicología y las ciencias sociales. En particular, tanto la definición de las especificaciones de producto como la interacción de los sistemas con los usuarios requieren de investigación psicológica y social. Así, partes de la psicología, como la psicología de la atención, han evolucionado en relación con el diseño de aparatos electrónicos.

En nuestra disciplina destaca la investigación psicológica realizada en interfaces de usuario y más concretamente sobre algunos aspectos de la usabilidad en condiciones experimentales. Un ejemplo muy reciente en nuestro país son las investigaciones realizadas sobre eye-tracking, por Marcos y González-Caro (2010) y Hassan, Herrero y Guerrero (2010).

\section{Destaca la investigación psicológica rea- lizada en interfaces de usuario y sobre algunos aspectos de la usabilidad}

La ingeniería de los sistemas de información y, en particular, de los servicios de información en la World Wide Web se está beneficiando también de la investigación psicológica. Un ejemplo muy importante es la aplicación del concepto del ciclo de vida - procedente de la psicología evolutivapara organizar la arquitectura de información de los servicios web municipales. La consideración del ciclo de vida es también fundamental cuando se abordan usuarios especiales, por ejemplo niños y adolescentes (García-Marco, 2009). Creemos que el modelo del ciclo de vida y la psicología diferencial van a ser cada vez más importantes para organizar de manera coherente el problema de la atención a los usuarios con características diferentes.

\section{Recuperación de la información}

La recuperación de la información se caracteriza por el diseño de sistemas para buscar de manera efectiva -de ahí recuperar-y eficiente. Incluye las listas ordenadas y los procesos realizados sobre ellas para mejorar su efectividad y eficiencia mediante modelos matemáticos de la información en los textos y las colecciones -modelos vectoriales y probabilísticos- o mediante el desarrollo de esquemas conceptuales que permitan su visualización y la navegación.

En este campo, se viene postulando desde hace más de dos décadas la importancia de ampliar los modelos de estudio con la incorporación de la psicología cognitiva -conocidos como modelos cognitivos (Ellis, 1992). Efectivamente, se ha avanzado mucho en dicho modelado, aunque lo cierto es que la práctica en la recuperación ha seguido siendo guiada fundamentalmente por modelos matemáticos y terminológicos del contenido lingüístico de los documentos y las colecciones. Aunque ya existen prototipos concretos, en general los sistemas que utilizan estos modelos -denominados interfaces inteligentes- siguen en fase de experimentación (Meadows, 2008, p. 407).
La definición y modelado de contextos y tareas es quizá la parte más importante de la contribución del paradigma cognitivo en recuperación de la información. A ello contribuye de forma muy notable el comportamiento informacional-information behaviour, en inglés. La contribución más importante de esta área, muy importante también por su aplicación a la alfabetización informacional, son los denominados modelos de búsqueda de información de autores como Case, Wilson, Kuhlthau o Dervin. La investigación sobre estos modelos está siendo abordada más bien mediante las metodologías de investigación-acción, que son también comunes en otras ciencias sociales aplicadas; por ejemplo desde la teoría de la actividad (Wilson, 2006).

La investigación sobre esquemas conceptuales para la navegación y la búsqueda no suele tener carácter experimental. Los modelos lingüísticos que sustentan el análisis terminológico, aunque tienen en cuenta lógicamente la pragmática y la semántica, no suelen requerir modelos experimentales, porque el significado de los signos lingüísticos por su propio carácter social es accesible de forma inmediata -con mayor o menor nivel de expertitud, claro está- para toda la comunidad de hablantes. Por ello, la investigación en organización del conocimiento raramente tiene carácter experimental, aunque el campo para ello podría ser muy amplio, especialmente si se atiende a su uso real, no a su diseño intelectual.

\section{Estructura y dinámica de la información}

Por encima del comportamiento individual se sitúa el comportamiento social. Se ha discutido mucho sobre su relación. Lo cierto es que ambos tienen entidad propia -no se pueden reducir completamente el uno al otro- y se determinan mutuamente, no de forma lineal.

El estudio del comportamiento social en torno a la información tanto a nivel micro como macro constituye un ámbito apasionante, pero todavía más complejo, si cabe, que el psicológico, pues se detectan en él múltiples niveles de estudio: relaciones de género, familiares, grupales, comunitarias, de clase social, económicas, políticas, culturales... El ser humano se clasifica -y parejamente elude también clasificaciones- en todos estos ámbitos, por lo que todos ellos son relevantes para explicar diferentes aspectos del comportamiento informacional.

Descendiendo a identificar áreas concretas de práctica e investigación, hay que decir que la bibliometría ha sido la que probablemente más evolucionado, ligada al esfuerzo del Estado por dirigir la investigación, a la que se identifica acertadamente como un factor clave del desarrollo económico. En España la investigación bibliométrica ha tenido tradicionalmente un lugar destacado, y ese esfuerzo está dando sus frutos actualmente con grupos de investigación de impacto internacional como Scimago, dirigido por Félix De Moya, o el Laboratorio de cibermetría, dirigido por Isidro F. Aguillo, que destacan por la aplicación de métodos y perspectivas muy innovadores, sin olvidar a otros grupos muy importantes en Granada, Salamanca, Valencia y otras sedes de investigación españolas.

Más dispersos han sido los esfuerzos por investigar la estructura económica y política de las industrias e institucio- 
nes de la información y el uso de los productos y servicios de información de una manera sistemática, a pesar de que se cuenta con buenas estadísticas públicas $y$, dado que los procesos de información están muy automatizados, de excelentes bancos de datos, que apenas sólo de vez en cuando afloran en la investigación. Aquí también se producirán en el futuro aportaciones importantes, siendo un campo de investigación que tiene que cuajar en un esfuerzo sistemático. Existen claro está iniciativas señeras de carácter parcial como el Observatorio la Lectura y el Libro del Ministerio de Cultura, entre otras. Recientemente, investigadores de departamentos de una decena de universidades españolas e iberoame-

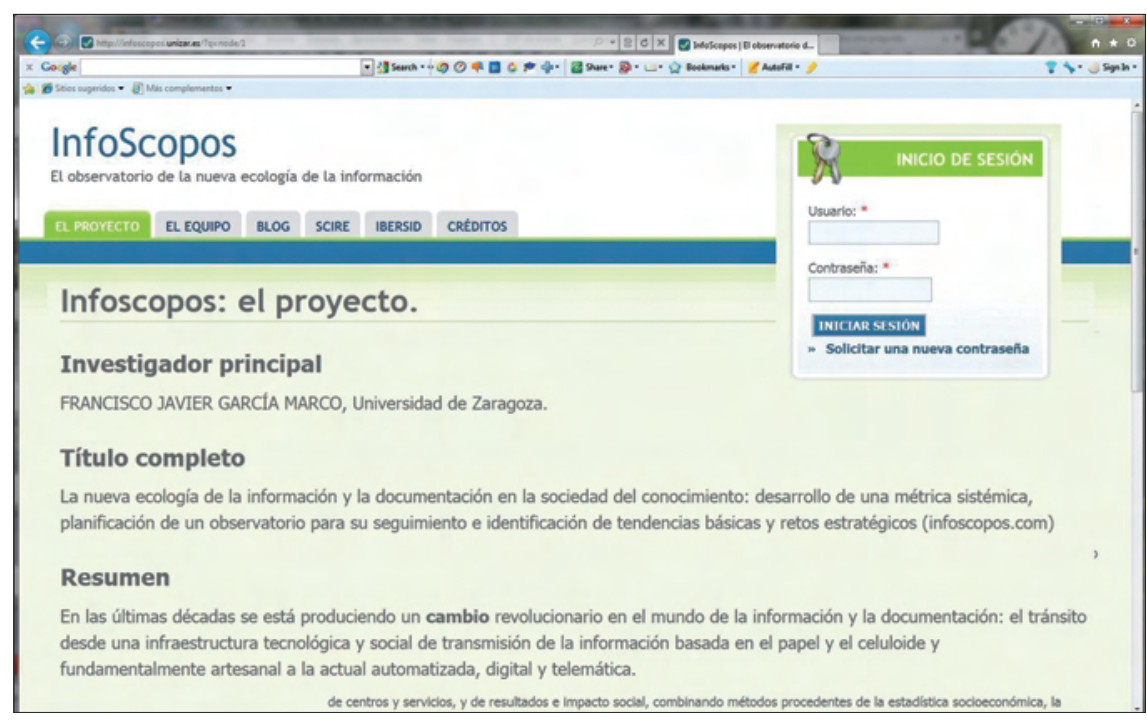

http://infoscopos.unizar.es ricanas se han organizado para desarrollar un observatorio centrado en monitorear el proceso de cambio digital en el ámbito de la información y la documentación, denominado Infoscopos, que pretende realizar un enfoque transversal.

\section{Teoría de la ciencia de la información}

La teoría de la información y la documentación está muy influida por la psicología y las ciencias sociales. En general, se acepta que la ciencia de la información es una ciencia social de carácter aplicado, que está a su vez encuadrada en los estudios sobre la información que se producen en todas las disciplinas desde la física, pasando por la bioquímica, la genética y la neurología, hasta llegar a la psicología, las ciencias sociales y la filosofía, y que se pueden visualizar como una estructura vertical, por niveles. No hay tanto acuerdo en nuestro país a la hora de asignarla a las humanidades o las ciencias sociales, como muestra el hecho de que esté excepcionalmente asignada a dos campos diferentes para la evaluación del profesorado universitario dentro de la Agencia Nacional de Evaluación de la Calidad y la Acreditación (Aneca).

Margaret Egan y Jesse Shera intentaron fundamentar el campo desde los estudios sociales, y definieron la bibliotecología científica como una epistemología social, y, más en concreto, como "el estudio de aquellos procesos mediante los cuales la sociedad como un todo busca conseguir una relación perceptiva y comprehensiva con su medioambiente integral -físico, psicológico e intelectual-" (Egan; Shera, 1952). Años más tarde, Shera ciñó ese objetivo tan ambicioso para redefinirla como aquella parte de la epistemología que estudia la manera en que el conocimiento -registrado en documentos- influye en la sociedad (Shera, 1961, 1970; Furner, 2002; vid. etiam Hjorland, 2002). Proponían pues una perspectiva a la vez social y humanista, desde la filosofía.

Una perspectiva muy fuerte desde el punto de vista social ha sido lógicamente la marxista, que en el campo más concreto de la ciencia de la información ha tenido un impacto especial a través de la psicología constructivista social, formulada por Vigotski y sus discípulos. Esta línea mantiene además contacto con los esfuerzos que hemos examinado anteriormente para acercar el campo de la recuperación de la información a las ciencias cognitivas y sociales.

La bibliotecología científica es la parte de la epistemología que estudia la manera en que el conocimiento -registrado en documentos- influye en la sociedad (Shera, 1961)

En general, la perspectiva social de la ciencia de la información está afectada por el propio carácter preparadigmático -en el sentido de que existen numerosos paradigmas alternativos en competencia- que caracteriza a las ciencias sociales, donde hay acuerdos sobre los temas a estudiar, pero más raramente respecto a los modelos teóricos y metodológicos. La propia orientación contemporánea de la ciencia a la resolución de problemas agrava el problema, pues disuade los esfuerzos de integración y reflexión teórica, aunque resulta muy fructífera en los microdominios estudiados.

Para rematar la complejidad de la perspectiva social en ciencia de la información hay que tener en cuenta la denominada crisis de las ciencias sociales, enmarcada en la más amplia crisis de la modernidad, que ha desembocado en el conjunto de las denominadas perspectivas posmodernas, que lógicamente han tenido también su traslación al campo de la información -una aproximación se puede consultar en el trabajo de Cronin (2008) ya citado.

Además de estos enfoques, han aparecido recientemente nuevos esfuerzos de integración científica que tienen todavía un carácter emergente. Dentro de estas nuevas perspectivas de convergencia entre ciencias humanas, naturales y tecnológicas destacan por su empuje las socioevolucionistas (v. g. Bates, 2005) y sociocibernéticas.

Finalmente, es importante señalar que la reflexión sobre la información como fenómeno humano no puede limitarse a las cuestiones que por analogía con otros modelos científicos disponibles y por la disponibilidad de métodos se pue- 
den tratar científicamente. El mundo de la información es un campo de la actividad personal y social en el que continuamente se tienen que tomar decisiones, y raramente es posible esperar a disponer de evidencia y a controlar todos los aspectos lógicos, metodológicos y teóricos. Estas decisiones no se toman, sin embargo, al margen de la racionalidad. Por el contrario, los aspectos éticos, culturales y estéticos de la actividad informacional son objeto de la reflexión y acción individual, de la opinión y la actividad pública y, en lo que se refiere a la reflexión colectiva sobre estos problemas, de las humanidades, y estos ámbitos son a posteriori el dominio real que estudia la ciencia de la información como disciplina que quiere ser científica pero que no puede abordar la totalidad de la realidad de la información.

\section{Los aspectos psicológicos y sociales son} omnipresentes en el trabajo y la reflexión de los profesionales de la información

\section{Conclusiones}

Los aspectos psicológicos y sociales son omnipresentes en el trabajo y la reflexión de los profesionales de la información. $\mathrm{Y}$, sin embargo, muchas veces se dejan de lado por su complejidad, que frecuentemente resulta desbordante.

A pesar de ello, en los últimos años la ciencia de la información está realizando un tránsito difícil desde una conceptualización abstracta o personalista del usuario y una focalización en herramientas informáticas genéricas para abordarlo como un sujeto cuyas necesidades informacionales, su comportamiento informacional y su sentido de la relevancia pueden ser entendidos mejor desde sus características psicológicas y sociales.

De alguna manera podríamos afirmar, por un lado, que la perspectiva social disuelve al usuario como ente abstracto y compacto y lo recupera como miembro de un entramado de dimensiones sociales, si se quiere, de grupos de usuarios; y que la perspectiva psicológica concreta al usuario como un individuo con una posición peculiar en el ciclo de vida y con unas diferencias que deben ser atendidas. Por el otro, ambas perspectivas permiten construir modelos del ser humano más complejos y ricos, y por tanto superar los esquemas demasiado simplificados que son de uso frecuente en nuestra disciplina.

La comprensión del usuario se vuelve así mucho más analítica y nos permite abordar el reto de la creciente personalización de los productos y servicios de información que exigen los usuarios, y que constituye una de las tendencias clave en el campo de la información.

Pero para hacer todo esto bien es necesario ser prácticos, estar al día y recorrer el camino de vuelta. Ser prácticos, sin descuidar -en aras de la interdisciplinariedad y la orientación a los problemas concretos- los nichos profesionales en los que existen los profesionales de la información. Estar al día, sin conformarnos con modelos de otras disciplinas que ya han quedado obsoletos. Y recorrer el camino de vuelta, comunicando nuestros hallazgos relevantes también en la otra dirección, hacia las disciplinas de las que nos nutrimos, para ganar relevancia y visibilidad dentro del conjunto de las ciencias, pero sobre todo porque es la única manera de contribuir al avance de la ciencia como un todo y de insertarse en el proyecto científico contemporáneo.

\section{Agradecimientos}

Este artículo ha sido producido en el marco del proyecto CSO2009-0761 subvencionado por el Ministerio de Ciencia e Innovación.

\section{Referencias}

Bates, Marcia J. "Information and knowledge: an evolutionary framework for information science". Information research, 2005, v. 10, n. 4.

http://informationr.net/ir/10-4/paper239.html

Cronin, Blaise. "The sociological turn in information science". Journal of information science, 2008, v. 34, n. 4, pp. 465475.

Egan, Margaret E.; Shera, Jesse H. "Foundations of a theory of bibliography". Library quarterly, 1952, v. 22, pp. 125-137.

Ellis, David. "Paradigms and proto-paradigms in information research". En: Vakkari, P.; Cronin, B. (eds.). Conceptions of library and information science: historical, empirical and theoretical perspectives. London: Taylor Graham, 1992, pp. 165-186. ISBN 0947568522.

Furner, Jonathan. "Shera's social epistemology recast as psychological bibliology". Social epistemology, July 2002, v. 16, n. 1, pp. 5-22.

García-Marco, Francisco-Javier. "Bibliotecas digitales para niños y adolescentes: psicología del desarrollo y diseño de sistemas de información". Ibersid: revista de sistemas de información y documentación, 2009, v. 3, pp. 247-254.

Hassan-Montero, Yusef; Herrero-Solana, Víctor; Guerrero-Bote, Vicente. "Usabilidad de los tag-clouds: estudio mediante eye-tracking". Scire, 2010, v. 16, n. 1, pp. 31-41. Número monográfico sobre 'Usabilidad: más allá del concepto', coord. por Francisco-Javier Martínez-Méndez.

Marcos, Mari-Carmen; González-Caro, Cristina. “Comportamiento de los usuarios en la página de resultados de los buscadores. Un estudio basado en eye tracking". El profesional de la información, 2010, julio-agosto, v. 19, n. 4, pp. 348- 358.

Meadows, Jack. "Fifty uears of UK research in information science". Journal of information science, 2008, v. 34, n. 4, pp. 403-414.

Shera, Jesse H. "Social epistemology, general semantics, and librarianship". Wilson library bulletin, 1961, v. 35, pp. 767-70.

Shera, Jesse H. Sociological foundations of librarianship. New York: Asia Publishing House, 1970. ISBN 0210222832.

Wilson, Tom D. "A re-examination of information seeking behaviour in the context of activity theory". Information research, 2006, v. 11, n. 4, paper 260.

http://InformationR.net/ir/11-4/paper260.html 


\section{Una Propesión. un fukuro}

$\triangle$

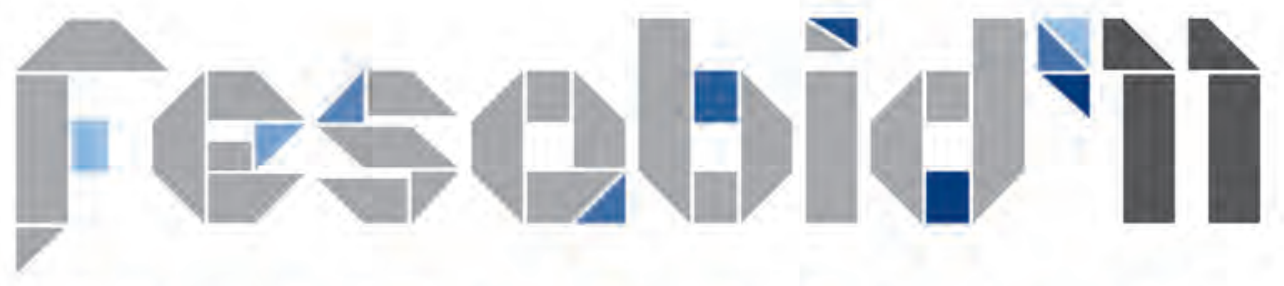

XII Jornadas Españolas de Documentación EBLIDA-NAPLE Conference 2011

in Cooperation with FESABID

XVI Jornadas Bibliotecarias de Andalucia

Una cita imprescindible para estar al día y evolucionar al ritmo cambiante de nuestra realidad profesional

http://www.fesabid.org/malaga2011

\section{Málaga, 25/26/27 Mayo 2011}

